\title{
Das Herz von Frédéric de Wangen, Bischof von Basel, 1776-1782
}

Walter Weber ${ }^{1}$

\section{Summary}

A leaden heart-shaped case with the year 1782 engraved, was found in the church St. Pierre in Porrentruy, Switzerland. It contained a preserved heart. Preservation had been obtained with vegetable materials and was such that microscopic and electron-microscopic preparations could be made. It was the heart of the bishop of Basel, Frédéric de Wangen. The heart is studied anatomically, and the historical background of the find is presented.

\section{Zusammenfassung}

In der Kirche St. Pierre in Pruntrut/Porrentruy fand man eine herzförmige Bleikapsel mit der Jahrzahl 1782, die das konservierte Herz eines Menschen enthielt. Die Konservierung war vornehmlich mit verschiedenen Pflanzenteilen erreicht worden und war so gut, dass man brauchbare mikroskopische und elektronenoptische Präparate herstellen konnte. Es handelte sich um das Herz von Frédéric de Wangen, Bischof von Basel. Das Herz wird anatomisch beschrieben, und es wird versucht, die kulturgeschichtliche Bedeutung des Fundes darzustellen.

Der Boden der Kirche St. Pierre in Pruntrut/Porrentruy ist mit Platten aus Jurakalk ausgelegt. Eine Platte vor dem Altar trägt ein Relief, das ein Herz darstellt, hinter ihm gekreuzt Bischofsstab und Schwert, darüber die Mitra mit Kreuz (Abb. 1).

1 Ich danke dem «Conseil de la commune ecclésiastique de Porrentruy» für die Überlassung des Präparats zu diesen Studien und für seine Geduld bei den vielen Verzögerungen.

Dr. h.c. Walter Weber, Anatomisches Institut, Bühlstrasse 26, Postfach, CH-3000 Bern 9 


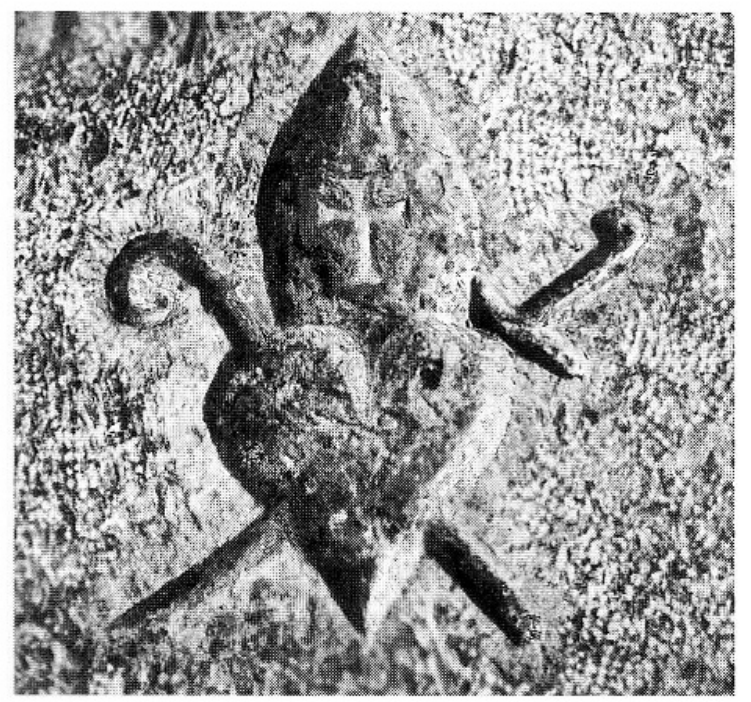

Abb.1. Relief der Bodenplatte vor dem Altar.

1980 mussten bei Bauarbeiten diese Platten zum Teil entfernt werden, auch diejenige mit dem Relief. Darunter kam in einem kleinen ausgesparten Raum eine Bleikapsel in Form eines stilisierten Herzens zum Vorschein. Sie misst an den breitesten Stellen $20 \times 20 \mathrm{~cm}$ und ist $6 \mathrm{~cm}$ dick. Die Kapsel ist ringsum verlötet und durch aufgelötete, gekreuzte Eisenbänder verstärkt. Die gewölbte Vorderseite trägt, durch eingeschlagene Punktreihen dargestellt, die Jahrzahl 1782 (Abb. 2). Die Hinterseite ist flach.

Durch Einschneiden wurde die Hinterwand der Kapsel sorgfältig ihrem Rand entlang geöffnet und aufgeklappt. Es befand sich darin eine dunkelbraune, feuchte Masse mit rauher Oberfläche und von undefinierbarem, unangenehmem Geruch. Bei genauem Hinsehen erkannte man an der Oberfläche eindeutig Pflanzenteile, z.B. Bruchstücke von Lorbeerblättern.

Die locker aufliegende, etwa $1 \mathrm{~cm}$ dicke Pflanzenschicht wurde sorgfältig abgestreift und es blieb ein ungefähr $2 \mathrm{~cm}$ dicker, elastischer Körper zurück. Nach vorsichtigem Waschen, erkannte man ein zwar stark geschrumpftes, aber relativ gut erhaltenes Herz. Trotz vieler Runzeln und Falten konnte man sich orientieren, die Flächen zuordnen, die Gefäss-Stämme bestimmen.

Bei beiden Kammern waren je zwei längsgestellte Einschnitte zu finden, die gegen die Herzspitze zusammenliefen, also eine V-Form bildeten. Seit 1877 werden Herzen zu diagnostischen Zwecken nach den Angaben von R.Virchow ${ }^{2}$ eröffnet, in der Verlaufsrichtung des Blutes, wobei auch die Vorhöfe eingeschnitten werden, was in unserem Fall nicht geschehen ist.

2 R. Virchow, Die Sektionstechnik im Leichenhaus des Charité-Krankenhauses. 2. Aufl. Berlin 1877. 


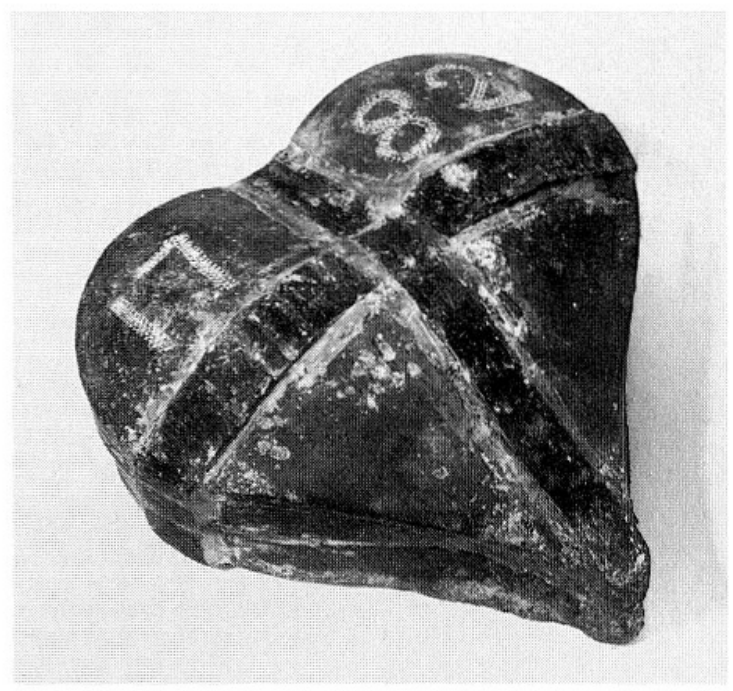

Abb. 2. Die Bleikapsel.

Nachdem in den Binnenräumen des Herzens keinerlei Pflanzenreste vorhanden waren, dürfen wir wohl annehmen, dass die Einschnitte nicht zur besseren Konservierung erfolgten, sondern aus diagnostischen Gründen, in einer einfacheren Schnittführung, als es heutzutage geschieht.

Die Einschnitte gaben aber den Blick in die Herzkammern frei, und wir stellten fest, dass auch die Innenoberflächen, die Klappen, Papillarmuskeln und Sehnenfäden gut erhalten waren.

Alle erkennbaren Formelemente und Strukturen liessen den Schluss zu, dass es sich eindeutig um das Herz eines Menschen handelte.

Zur Anfertigung mikroskopischer Präparate wurden einige kleine Stücke entnommen ${ }^{3}$. Auch im mikroskopischen Bereich stellten wir eine erstaunlich gute Erhaltung des Gewebes fest. Zwar liessen sich die Schnittpräparate nicht so gut anfärben, wie wir es bei heutigem Material gewohnt sind, aber man konnte die Querstreifung der Muskelfasern deutlich erkennen (Abb. 3a).

An vielen Stellen fand man das sogenannte Abnutzungspigment (Lipofuszin), das in der Herzmuskulatur mit zunehmendem Alter gewöhnlich auftritt.

Bei Menschen über 40 Jahren entsteht eine typische Veränderung der Herzmuskulatur in Form einer Dickenzunahme der Muskelfasern. So messen wir bei unserem Beispiel (Abb. 3a) einen Durchmesser von $50 \mu \mathrm{m}$, während für junge Menschen 10-20 $\mu \mathrm{m}$ angegeben werden. Bei Sportlern

3 Nach Einbetten in Paraffin wurden $10 \mu \mathrm{m}$ dicke Schnitte hergestellt und diese mit «saurem Hämatoxylin nach Ehrlich» gefärbt. Ich danke Frau A. Lieglein für die Herstellung der mikroskopischen Präparate. 


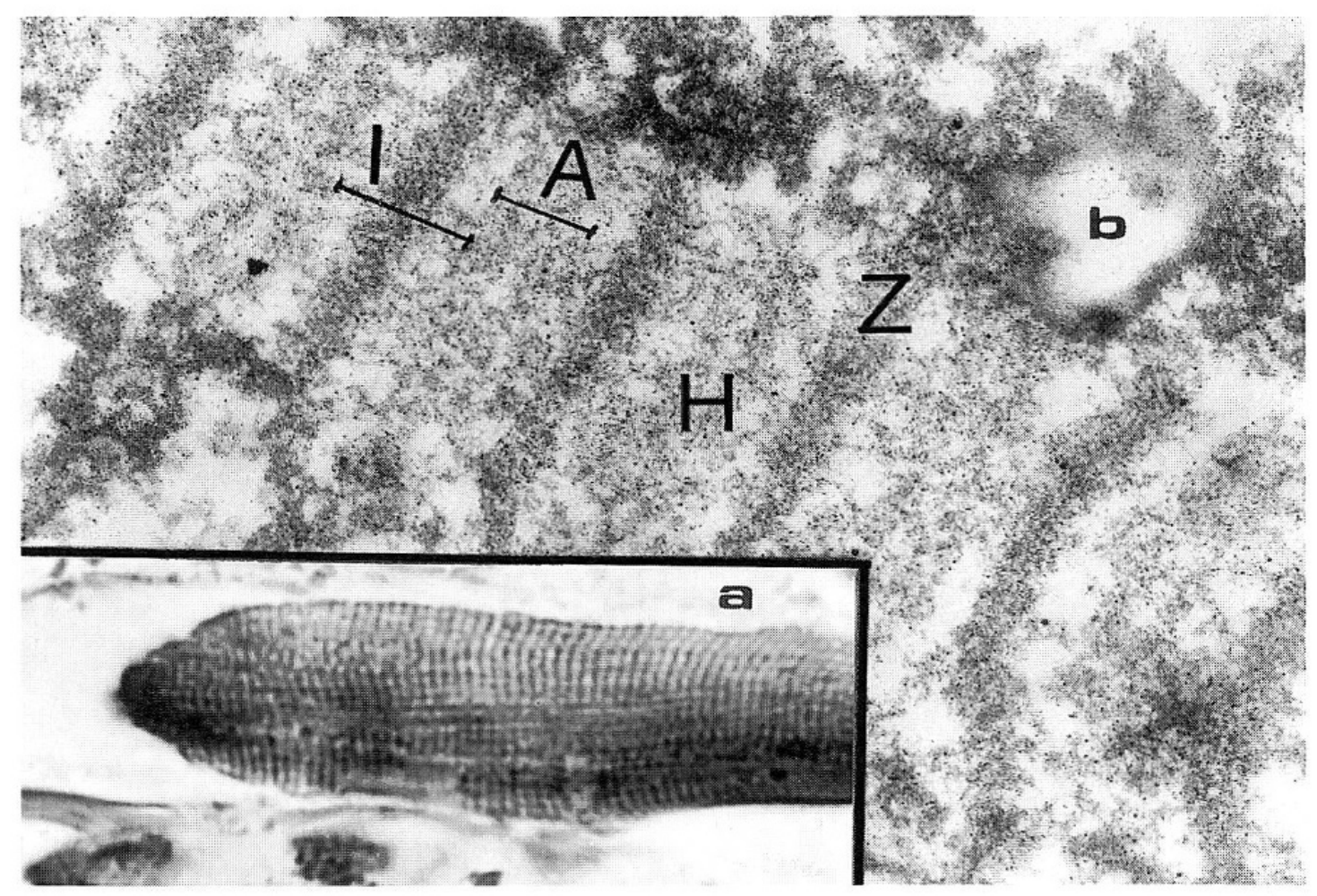

Abb. 3. Ausschnitt 3a: Muskelfaser mit Querstreifung (Lichtmikroskop), Vergrösserung 400×. 3b: Elekronenmikroskopische Aufnahme Z-, H-, I- und A-Streifen, Vergrösserung: $10000 \times$.

kann sich die Blutversorgung durch gleichzeitige Vermehrung der Kapillaren anpassen, bei weniger tätigen Menschen kommt es wegen der geringeren Diffusion durch die verdickten Fasern zu einer Verminderung der Nährstoff- und Sauerstoffzufuhr, sowie zu einem verzögerten Abtransport von Stoffwechselprodukten. Daraus resultiert eine Schädigung der Muskelfasern, was schliesslich zu deren Abbau führen kann, wobei der Raum durch Binde- und Fettgewebe eingenommen wird ${ }^{4}$.

So fanden wir denn zwischen den Muskelfasern und unter dem Epikard (Oberflächen-Überzug des Herzens) wechselnde, relativ grosse Mengen von Fett-Einlagerungen. Bei der Verarbeitung solcher Gewebe zu mikroskopischen Präparaten wird das Fett in der Regel herausgelöst. In unserem Fall hat sich aber das Fett mit dem Kalk des Wassers verbunden zu sogenannter Kalkseife, welche in den üblichen Lösungsmitteln nicht löslich ist. Sie hat bestimmte optische Eigenschaften und kann im Lichtmikroskop bei sogenannter Dunkelfeldbeleuchtung und im polarisierten Licht eindeutig als Kalkseife nachgewiesen werden. In Porrentruy dürfte das Brunnenwasser

4 F. Büchner, Allgemeine Pathologie. 4. Aufl. München-Berlin 1962.

Herrn PD. Dr. A. Stofer vom Gerichtlich-medizinischen Institut Bern danke ich für die Beurteilung der mikroskopischen Präparate. 


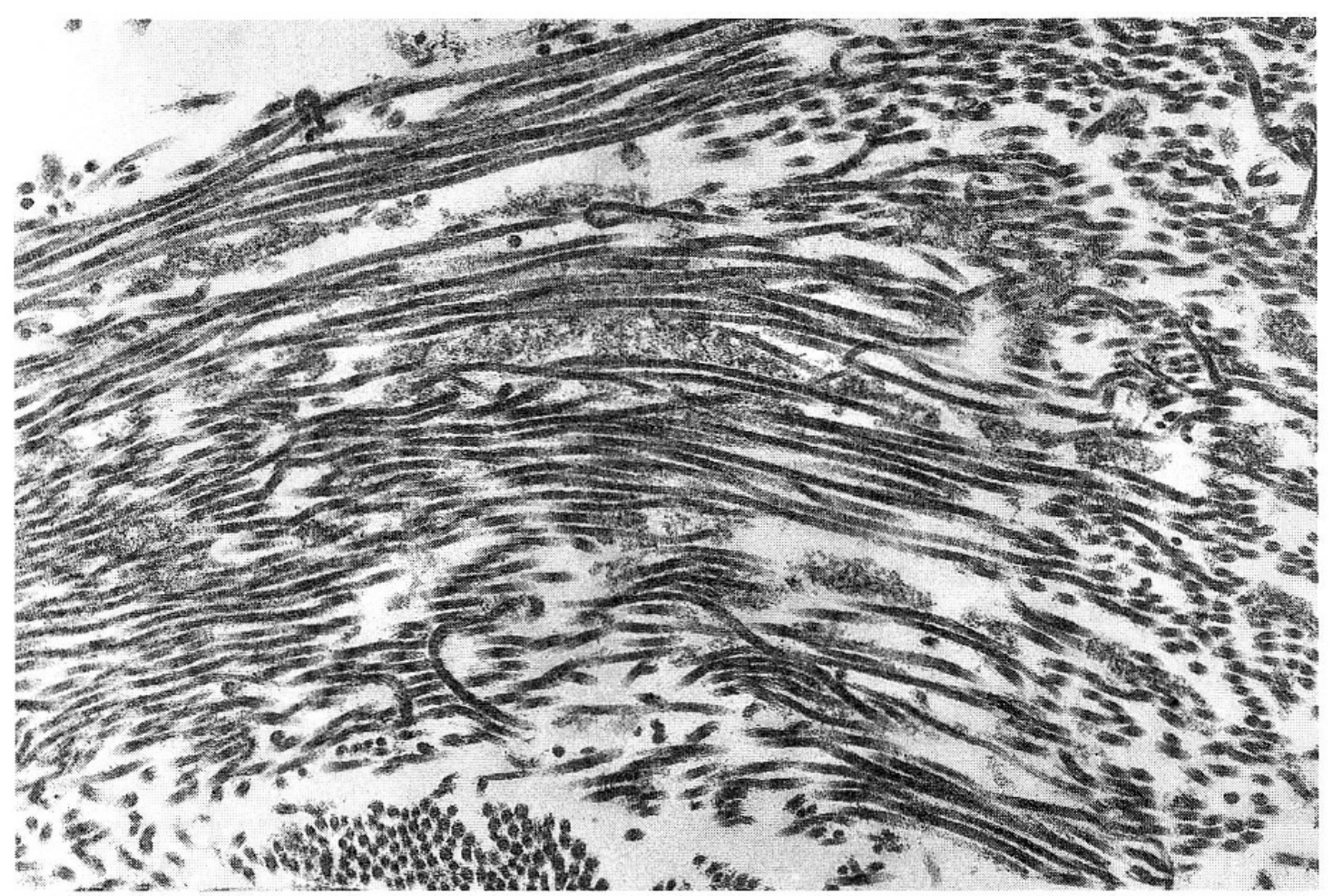

Abb. 4. Fibrillen des kollagenen Bindegewebes. Querstreifungs-Perioden $64 \mathrm{~nm}$. Vergrösserung: $36000 \times$.

schon damals einen hohen Kalkgehalt gehabt haben ${ }^{5}$. Sehr wahrscheinlich wurde das Herz vor der Konservierung längere Zeit gewässert, denn einmal in der Bleikapsel, konnte sicher nicht mehr die nötige Wassermenge eindringen. Die Bleikapsel muss vollkommen dicht gewesen sein, sonst wäre das Präparat längst ausgetrocknet.

Selbst im Elektronenmikroskop liessen sich - wenn auch stark verändert - die Grundformen der Muskulatur erkennen. Bezeichnenderweise hatte sich der sogenannte Z-Streifen am besten erhalten, der aus komplex vernetzten und dadurch besonders widerstandsfähigen Proteinmolekülen besteht. Die Aufhellung des H-Streifens ist mehr oder weniger deutlich sichtbar, sogar die feinen A- und I-Bänder sind einigermassen zu erkennen (Abb. 3b), das Abnützungspigment Lipofuszin war eindeutig bestimmbar. Die Fibrillen des kollagenen Bindegewebes zeigten sogar die bekannte Periodizität und unterschieden sich nicht wesentlich von entsprechenden rezenten Präparaten $(\mathrm{Abb} .4)^{6}$.

5 Das Laboratoire des Eaux, St-Ursanne teilt uns mit, dass die gegenwärtige Wasserhärte in Porrentruy $30^{\circ} \mathrm{fH}$ beträgt.

6 Herr Prof. O. Müller am Anatomischen Institut Bern hat die elektronenmikroskopische Bearbeitung übernommen, wofür ich ihm herzlich danke. 
Wie kommt es, dass dieses Herz so gut erhalten ist? Erst 1832 wurden Mittel bekannt, die Gewebe und Organe so zu konservieren, dass die mikroskopischen Strukturen erhalten bleiben und gut dargestellt werden können ${ }^{7}$.

Zur Untersuchung der Pflanzenschicht wandten wir uns an das Botanische Institut der Universität Bern. Hier der Bericht von Frau Dr. Ruth Schneider:

Die Leute ... machten einen grosszügigen Griff in die Gewürzdosen. Folgende Arten konnte ich bestimmen:

- Kümmel (Carum carvi): Samen und wenige Blätter

- Lavendel (Lavandula augustifolia): Samen und evtl. Blätter

- Salbei (Salvia officinalis): Blätter und evtl. ein Same

- Kamille (Matricaria chamomilla): 1/2 Blütenköpfchen

- Thymian (Thymus spec.): mehrere Blütenkelche

- Dost (Origanum vulgare): viele Blütenkelche und mehrere Blütenstände

- Lorbeer (Laurus nobilis): Blätter

- Rosmarin (Rosmarinus officinalis): viele Blätter

- Sevi (Juniperus sabina) ein kleines Zweiglein.

Nicht bestimmbar sind Farnreste, ein Gras, drei kleine Früchte, die Blüte eines Schmetterlingsblütlers (Papilionaceae) und einige Holzstückchen. Ich bin überzeugt, dass noch zwei bis drei weitere Kräuter dabei sein müssten, aber diese zu bestimmen ist der kleinen Überreste wegen nicht möglich. Was nun die Konservierung des Herzens bewirkt hat, kann ich nicht sagen. Ich möchte vermuten, dass alle diese Arten mit ihren starken ätherischen Ölen dazu beigetragen haben.» ${ }^{8}$

Für mehrere der bestimmten Pflanzen gibt $\mathrm{H}$. Braun ${ }^{9}$ ausser den ätherischen Ölen auch noch einen Gehalt an Gerbsäuren an. Diese Substanzen können direkt mit Proteinen reagieren und stabile Verbindungen eingehen, also eine Konservierung erreichen. Zu bestimmten Zwecken werden auch heute noch Tannin-haltige (Gerbsäure) Konservierungsmittel in der Elektronenmikroskopie angewandt.

Pflanzen mit ätherischen Ölen wurden schon von den alten Ägyptern zum Einbalsamieren verwendet. Die Leibeshöhlen wurden nach der Organentnahme mit Palmwein gewaschen und anschliessend mit solchen Pflanzen gefüllt. Primär hatten diese die Funktion, den Geruch zu korrigieren, wirkten

71812 fixierte J. Hill als erster pflanzliche Gewebe in Alaun und härtete sie in Alkohol, um sie schneiden zu können. 1832 verwendete L. L. Jacobson Alkohol, Sublimat und Chromsäure zur Fixierung tierischen und menschlichen Gewebes. 1893 publizierte F. Blum die erste Arbeit über die Anwendung von Formaldehyd zu Fixationszwecken, das von da an in verschiedenen Mischungen das wichtigste Fixationsmittel wurde (E. Hintzsche, Die Entwicklung der histologischen Färbetechnik, Ciba Zeitschrift Nr. 88,1943) (B. Bracegirdle, A History of Microtechnique, London 1978).

8 Vielen Dank an Frau Dr. Ruth Schneider für die präzise Auswertung der Pflanzenüberreste.

9 H. Braun, Heilpflanzen-Lexikon, Fischer Taschenbuch Verlag, Frankfurt am Main 1971. 
aber ausserdem konservierend. Die entnommenen Organe wurden in Tonkrügen in Salzlösungen aufbewahrt. Die betreffenden Salze konnten aus den sogenannten Sodaseen in Ägypten gewonnen werden. Ähnlich wurde in unseren Breiten bis nahe an unsere Zeit verfahren ${ }^{10}$.

Der Chemiker J.-N. Gannal zählt in seiner gründlichen Arbeit nach Literaturangaben eine lange Reihe von Pflanzenmischungen auf, die bei Einbalsamierungen in Europa verwendet wurden, wobei auch die Pflanzen unserer Mischung in verschiedenen Kombinationen vorkommen ${ }^{11}$.

Man kann also festhalten, dass die Konservierung des Herzens mit damals gebräuchlichen Mitteln durchgeführt wurde. Leider fanden wir keine Hinweise auf die Person, die die nötigen Vorkehrungen getroffen hatte.

Gannal empfiehlt schliesslich zur Konservierung, speziell für anatomischen Gebrauch, Alaun und Aluminiumazetat ${ }^{11}$.

Um 1840 wurden für makroskopische Arbeiten Arsen- und Schwermetallsalze eingeführt. Wir fragten uns, ob beim Herzen, vielleicht als eine Art Pioniertat, schon solche Stoffe verwendet worden seien. Darum liessen wir das Wasser, in dem wir das Herz ausgewaschen hatten, nach verschiedenen Stoffen untersuchen. Das Kantonale Laboratorium und das Chemielabor des Gerichtlich-medizinischen Instituts ${ }^{12}$ haben für uns Untersuchungen angestellt zum Nachweis von Schwermetallen und bestimmten Salzen, die aber praktisch alle negativ verliefen. Einzig das Blei war etwas erhöht, was wohl mit der Aufbewahrung in der Bleikapsel zusammenhängt.

Man fand immerhin Chlorid- und Sulfat-Ionen. Dass Kochsalz zugefügt worden war, ist nicht weiter verwunderlich. Beim Sulfat konnten wir durch Reaktion mit Hämatoxylin nachweisen, dass es sich um Alaun handelte. Da Alaun in der Natur als Alaunstein (Alunit) vorkommt, wurde seine Wirkung schon in vorchristlicher Zeit bekannt und bei der Ledergerbung angewandt.

Bei unserem Herzen ist sicher die Verwendung von Alaun die Erklärung der guten Erhaltung des kollagenen Bindegewebes mit seinen Fibrillen.

Das Herz ist also recht gut konserviert. Es hat aber von den Pflanzen mit Gerbsäuren eine starke Braunfärbung angenommen, die es leicht an die Lösungen abgibt, in die es zur Aufstellung in heutiger Art, eingelegt werden muss.

Zur besseren Präsentation wurden die Einschnitte einigermassen zugenäht und die Räume, so gut es ging, mit Paraffin ausgegossen. So zeigt das Präparat wieder ungefähr die Gestalt des ursprünglichen Herzens (Abb. 5).

10 F. Paul, Die Leichenkonservierung, Ciba Zeitschrift Nr. 43, 1937.

11 J.-N. Gannal, Histoire des Embaumements, Paris 1838.

12 Besten Dank auch ihnen für ihre Arbeit. 


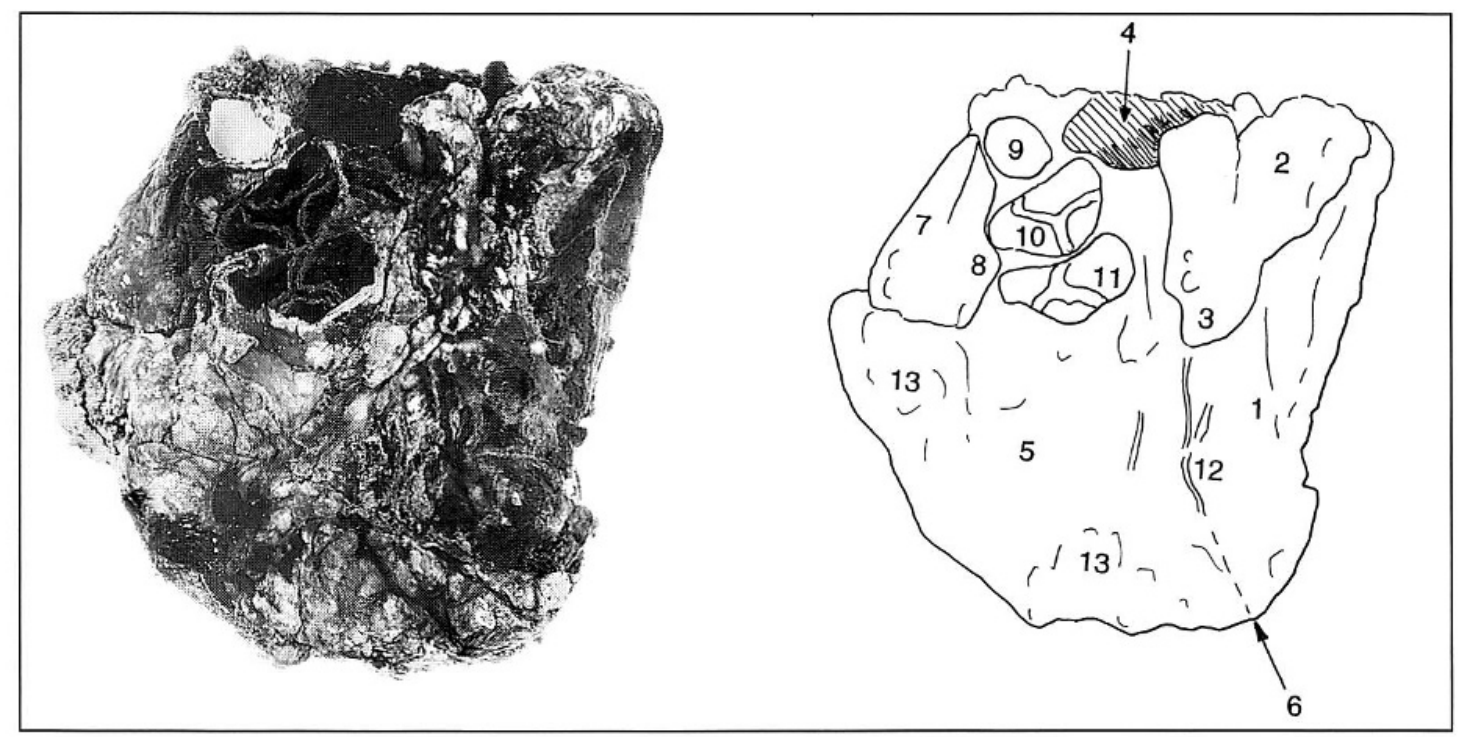

Abb. 5. Oberfläche der Vorderwand (nach Bearbeitung):

1 Vorderwand der linken Kammer;

2 Vorderwand des linken Vorhofs;

3 linkes Herzohr;

4 von oben eröffneter linker Vorhof;

5 Vorderwand der rechten Kammer;

6 Grenze zwischen linker und rechter Kammer, Pfeil an der Herzspitze;

7 Aussenwand des rechten Vorhofs;

8 rechtes Herzohr;

9 obere Hohlvene (mit Paraffin gefüllt);

10 Aorta mit Taschenklappe;

11 Lungenarterie mit Taschenklappe. (Im Leben sind die beiden Gefässe rund und glattwandig, die Klappen gespannt und nicht so faltig wie in unserem Präparat. Die beiden Herzohren füllen die Vertiefungen beidseits der Lungenarterien auf, was hier nur für das rechte zutrifft.)

12 Vorderer Zwischenkammerast der linken Kranzarterie (er liegt über der Kammerscheidewand, die Grenzen der Kammern an ihrer Oberfläche markierend);

13 Polsterfett, dem Alter entsprechend in normaler Menge.

Soviel zur anatomischen Beschreibung. Aber dem Herrn Bischof ging es natürlich nicht um die Erhaltung seines Herzens als interessantes anatomisches Präparat. Das Herz gilt seit jeher als Träger der Verbundenheit und Liebe. Man kennt eine ganze Reihe von Fällen mit gesonderter Beisetzung von Herzen. Ein Beispiel aus sehr früher Zeit ist Elisabeth die Heilige von Thüringen, gestorben 1231 in Marburg und daselbst auch beigesetzt, während das Herz nach Thüringen verbracht wurde. Dass es zu dieser Zeit, da Leichenöffnungen zu medizinischen Zwecken nicht gestattet wurden, möglich war, ein Herz zu entnehmen, spricht für die grosse symbolische Bedeutung, die diesem Organ beigemessen wurde.

Bekannt dürfte das «Herzgrüftel» in der Augustinerkirche in Wien sein, wo 54 Herzen von Mitgliedern des österreichischen Kaiserhauses in silber- 


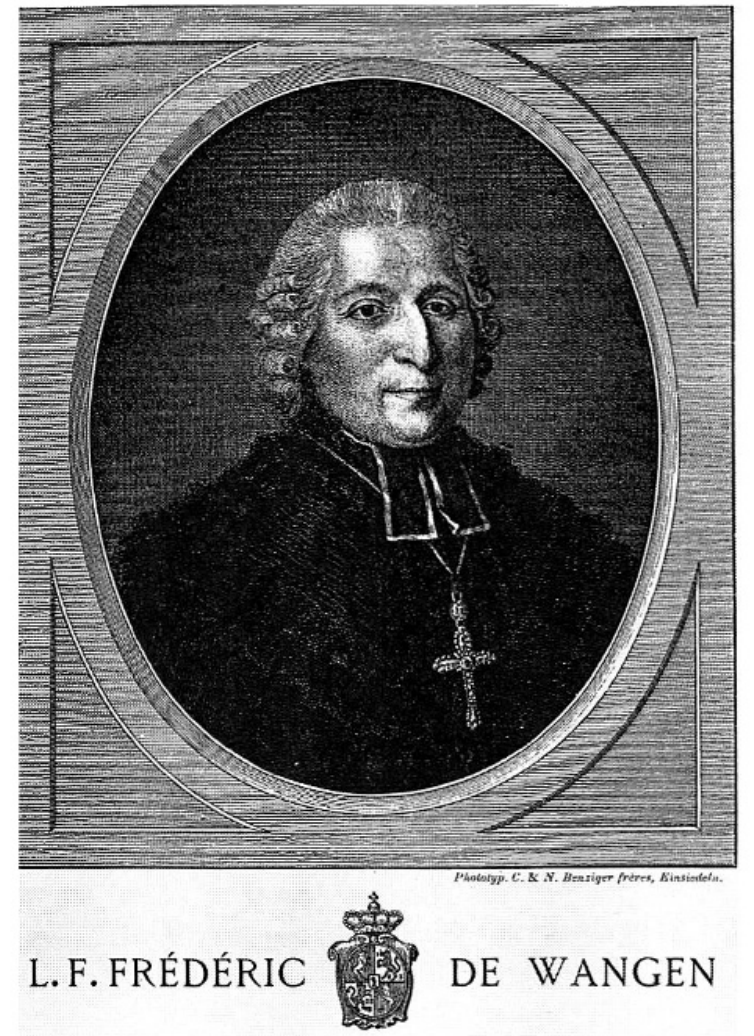

Abb. 6. Portrait von Herrn Bischof de Wangen (aus Vautrey) ${ }^{16}$

nen Behältern aufbewahrt werden. Die Herzen hat man bei der Einbalsamierung der Leichname entnommen. In der Kapuzinergruft befinden sich 141 Einbalsamierte, es sind also nicht alle Herzen gesondert beigesetzt worden ${ }^{13}$.

Wer war dieser Fürstbischof Frédéric de Wangen (Abb. 6) und was hat ihn bewogen, diese Herzbeisetzung zu verfügen? Er wurde geboren am 12. März 1727 in Wilvisheim in der Diözese Strassburg und auf den Namen Friedrich Ludwig Franz von Wangen von Geroldseck getauft. Seine Familie, ein altes Adelsgeschlecht im Unter-Elsass, brachte mehrere bekannte Würdenträger hervor. Kaiser Sigismund autorisierte die Familie im Jahre 1413, Namen und Wappen der «von Geroldseck» zu führen. Der Werdegang des Bischofs von Wangen wird kurz folgendermassen beschrieben: «Sein Aufstieg führte ihn vom Kanonikat zur Mitgliedschaft am Kapitel, zu Kustodie, Archidiakonat, Amt eines Kantors und gewähltem Bischof. Am 15.3. 1766 empfing er die Weihe des Priesters, am 3.3.1776 in Bellelay jene des Bischofs, nachdem der Papst die Wahl des Kapitels am 13.1.1775 bestätigt hatte.» ${ }^{14}$

13 L. C. Friedlaender, Wien. Verlag für Jugend und Volk, 6. Aufl. 1963.

14 Helvetica sacra I/1, herausgegeben von A. Bruckner, Erzbistümer A-Ch, Francke-Verlag, Bern 1972. 
Der Fürstbischof war bei allen Menschen, gleich welchen Standes, beliebt. Man nannte ihn «le beau prince», denn er feierte rauschende Feste und zog bei Besuchen in benachbarte Städte, wie etwa Biel oder La Neuveville, mit all seinen Hofleuten in glanzvollen Umzügen ein ${ }^{15}$.

Ein bestimmtes Verhältnis in seinem Reich beschäftigte ihn sehr. Seit 1528, dem Jahr der Reformation von Basel, lebte der Bischof von Basel im benachbarten Pruntrut, das aber, wie die ganze Ajoie, kirchlich dem Erzbischof von Besançon unterstellt war. In Porrentruy war der Bischof von Basel nur weltlicher Fürst, symbolisch dargestellt mit dem den Bischofsstab kreuzenden Schwert. Bischof blieb er für seine verbliebenen Gemeinden um Basel und im Elsass. Es war Frédéric de Wangens grösster Wunsch, das zu ändern. Und es gelang ihm! Sein gutes Verhältnis zu Versailles erleichterte offensichtlich die Verhandlungen zu einem grossen Abtausch von Gemeinden. Sein Weihbischof, Jean Baptist Gobel, Bischof von Lydda, bekannt für besondere Geschicklichkeit im Verhandeln, und der Vertreter unseres Fürstbischofs am königlichen Hof, Abbé de Raze, erarbeiteten mit Messire Pierre de Fraigne, Vertreter des Erzbischofs von Besançon, und den Bevollmächtigten des Königs einen Vertrag: 29 Gemeinden im Elsass und um Basel, alle zur Diözese Basel gehörig, wurden getauscht gegen 20 Gemeinden der Diözese Besançon, darunter Porrentruy selber. Damit wurde Bischof de Wangen auch geistlicher Oberhirte seiner näheren Umgebung. Der Vertrag konnte in Paris am 17. November 1779 unterzeichnet werden.

Anschliessend mussten noch die genauen Grenzen zwischen Frankreich und dem bischöflichen Gebiet nachkontrolliert und an einigen Stellen korrigiert werden. Dieser Beschluss wurde am 11. Juli 1780 unterzeichnet. Der «Tauschvertrag» wurde ratifiziert von Kaiser Joseph II., von König Louis XVI. und von Papst Pius VI. Das genaue Datum der Übergabe war schliesslich der 20. Januar $1781^{16}$.

Und noch ein dritter Vertrag zwischen Frankreich und Bischof de Wangen wurde abgeschlossen, auch diesmal ausgearbeitet von den beiden treuen Freunden Bischof Gobel und Abbé de Raze. Es handelte sich um die Erneuerung und Erweiterung eines Vertrags von 1739. Er enthielt in 16 Artikeln Bestimmungen über das gegenseitige Verhalten bei politischen und kriegerischen Auseinandersetzungen, gegenseitige Unterstützung, Neutralität der bischöflichen Lande bei Angriffen anderer Mächte gegen Frankreich; alle rechtlichen Belange des Zivil-, Obligationen- und Strafrechts wurden an-

15 P.-O. Bessire, Histoire du Jura bernois et de l'ancien Evêché de Bâle. Editions de la prévôté, Moutier 1977.

16 Mgr. L. Vautrey, Histoire des Evêques de Bâle, 2 vol. Einsiedeln, New York, Cincinnati, St. Louis $1884-1886$. 
geglichen; Zoll- und Handelsbestimmungen, z.B. über das Salz und andere Güter, wurden festgelegt. Der Vertrag war fertig ausgearbeitet in Versailles am 20.6.1780, er wurde ratifiziert vom Fürstbischof am 1.7., von König Louis XVI. am 11.7.1780.

All dies war nun wirklich eine ganz besondere Leistung des Bischofs von Basel für Porrentruy, und es wird uns verständlich, dass Bischof de Wangen für diese ihm am Herzen liegenden Verhältnisse ein spezielles Zeichen setzen wollte mit der Verfügung dieser Herzbeisetzung.

Es bleibt noch die Frage, unter welchen Bedingungen man das Herz dem Körper entnommen hatte. Die Antwort ist einfacher als erwartet. Wir lesen bei Vautrey ${ }^{16}$ :

«A l'ouverture du cadavre, on a trouvé le foie, la rate et l'estomac gangrenés, le cœur même était attaqué ainsi qu'un des poumons.»

Es war also ganz selbstverständlich, dass man eine Obduktion durchführen würde, der Bischof wusste es und konnte bestimmen, was mit dem Herzen geschehen sollte.

Über die Todesursache und die Krankheiten, unter denen der Bischof litt, ist aus den Berichten nichts Genaues zu entnehmen, denn unter «gangrène» kann sowohl Brand (Nekrose) als auch Fäulnis oder Krebs verstanden werden.Am Herzen fanden wir keine pathologischen Veränderungen, ausser den erwähnten Alterserscheinungen. Da uns die anderen erwähnten Organe nicht zur Verfügung standen, können wir uns über andere mögliche Erkrankungen nicht äussern. Es wird berichtet, er habe unter Skorbut gelitten, aber die beschriebenen Verwüstungen des Oberkiefers sprechen für schwerere Erkrankungen. Weiter unten heisst es bei Vautrey ${ }^{16}$ :

«A l'église paroissiale, le cœur du vénérable défunt fut inhumé par le curé au pied de l'autel. A l'église des jésuites le P. Vogelweid prononça l'oraison funèbre du prince qui fut ensuite déposé dans le caveau de ses prédécesseurs.»

Beim Umbau der Jesuitenkirche wurden vor einigen Jahren die Gebeine in die Krypta von St-Pierre übertragen und dort eingemauert.

Leider findet diese geschichtliche Episode ein Ende, das nicht nach dem Sinn unseres Bischofs ausfiel. Bischof de Wangen starb 1782. An seine Stelle wurde Joseph Sigismund von Roggenbach gewählt, dessen Sympathien den Deutschen galten. Die französische Revolution brach aus, dem Vertrag von 1780 entsprechend, hielten sich französische Truppen bereit, zum Schutz der bischöflichen Lande dort einzumarschieren. Bischof von Roggenbach ersuchte den deutschen Kaiser um Hilfe. Dessen Truppen erhielten das Durchmarschrecht durch baslerisches Gebiet; es kam zur Kriegserklärung, die fran- 
zösischen Truppen rückten in die Ajoie ein, Bischof von Roggenbach floh nach Wien ${ }^{17}$.

Es bildete sich ein Verfassungsrat, und schon 1792 wurde eine «freie und unabhängige Republik Raurachien» ausgerufen, die allerdings nur kurzen Bestand hatte. Es kam anschliessend nicht mehr zum Aufbau eines Bistums, wie es Frédéric de Wangen erarbeitet hatte. Seine Mühe ist schlecht belohnt worden. Der Bischof von Basel war von 1528 bis 1781 nur Fürst von Porrentruy, seinem Wohnsitz, dann etwa 10 Jahre Bischof der Ajoie, und schon zerfielen wieder alle Verträge, die Frédéric de Wangen erarbeitet hatte.

17 M. Schwander, Jura, Konfliktstoff für Jahrzehnte, Benziger Verlag, Zürich 1977. 